\title{
Three-Fluid Axisymmetric Equilibrium Model and Application to Spherical Torus Plasmas Sustained by RF Electron Heating
}

\author{
Akio ISHIDA, Akira EJIRI ${ }^{1)}$, Yuichi TAKASE ${ }^{1)}$, Naoto TSUJII ${ }^{1)}$, Hiro TOGASHI ${ }^{1)}$, \\ Yusuke YOSHIDA $^{1)}$, Takahiro SHINYA ${ }^{1)}$ and Shintaro TSUDA ${ }^{1)}$ \\ 10-18 Ikarashinishi, Nishi-Ku, Niigata 950-2161, Japan \\ ${ }^{1)}$ The University of Tokyo, Kashiwa 277-8561, Japan
}

(Received 16 July 2015 / Accepted 17 September 2015)

\begin{abstract}
Radiofrequency (RF) waves could be used for plasma current start-up in spherical torus (ST) reactors, where plasma formation and current drive without the ohmic heating solenoid is required. In such a plasma, the electrons can be represented by two temperature components, i.e. high-temperature low-density electrons and lowtemperature high-density electrons. In order to describe the equilibrium of such plasmas, we develop a three-fluid (two electron fluids and one ion fluid) axisymmetric equilibrium model with toroidal and poloidal flows. This model has been applied for the first time to a recent TST-2 discharge, and we have obtained an equilibrium which is consistent with experimentally observed results. It is found that (1) the toroidal current density and pressure are dominated by the high-temperature low-density electron (eh-electron) fluid and (2) the radial force balance for each fluid species is quite different, i.e. the ion fluid is confined by the electric force due to the negative electrostatic potential while the eh-electron fluid pressure gradient force is balanced by the Lorentz force (its toroidal current density times the poloidal magnetic field). These results are different from previous speculations.
\end{abstract}

(C) 2015 The Japan Society of Plasma Science and Nuclear Fusion Research

Keywords: solenoid-free spherical torus plasma, flowing two-electron temperature plasma, axisymmetric equilibrium, flowing three-fluid equilibrium model

DOI: $10.1585 /$ prr.10.1403084

\section{Introduction}

The multi-fluid formulation of axisymmetric flowing equilibria contains important physics which is missing from the one-fluid model [1-4]. The poloidal flow speed singularity of the two-fluid equilibrium equations differs markedly from the one-fluid model [5]. The poloidal flows exceeding the neoclassical theory prediction have been observed in several tokamaks [2]. Since the neoclassical theory neglects the two-fluid effect to determine poloidal magnetic field structure, it is interesting to apply the two-fluid model to solve this discrepancy. In a high-performance discharge in NSTX, it was found that the ratio of the local ion inertial length to the local scale length of the ion pressure gradient, $\ell_{\mathrm{i}} / L_{\mathrm{pi}}$, can be large except in the core region, while the ratio of the local ion gyroradius to the local scale length of the ion pressure gradient, $\rho_{\mathrm{i}} / L_{\mathrm{pi}}$ remains small in the entire domain $[3,4]$. This suggests that the two-fluid model should be used to describe such equilibrium with steep gradient because the ion inertial length, $\ell_{\mathrm{i}} \equiv c / \omega_{\mathrm{pi}},\left(c\right.$ is the speed of light and $\omega_{\mathrm{pi}}$ is the ion plasma frequency) is the intrinsic scale of the two-fluid model and the one-fluid model is valid only if $\ell_{\mathrm{i}} / L_{\mathrm{pi}} \ll 1$ [6].

The formation of a high performance plasma without the ohmic heating solenoid was accomplished by combining time varying vertical and shaping fields with several heating and current drive tools [7]. Such a technique is

author'se-mail:frclab@cocoa.ocn.ne.jp crucial especially for spherical torus (ST) plasmas in order to realize a commercial fusion reactor. Recently a full two-fluid equilibrium model $[3,4]$ was applied to describe a solenoid-free RF sustained ST plasma [8]. In the past equilibrium reconstruction of such a plasma has been carried out using the Grad-Shafranov formalism $[9,10]$. Since some electrons are driven resonantly by the RF wave, the electrons should have two temperature components, i.e. high-temperature low-density electrons and lowtemperature high-density electrons [11]. Recent measurement of the electron temperature and density in the TST-2 experiment reveals the existence of high-temperature lowdensity electrons which carry almost all of plasma current. In order to describe the equilibrium state of RF sustained ST plasmas accurately, we develop here a new axisymmetric three-fluid equilibrium model consisting of a high-temperature low-density electron component, a lowtemperature high-density electron component and an ion component.

Section 2 presents the formulation of the model which includes a nonlinear algebraic equation for the density ratio as well as four 2 nd order partial differential equations and some algebraic equations. Since this model has nine arbitrary surface functions, how to select these functions is important in practice. In Sec. 3 we apply the model to a recent TST-2 discharge \#115620 at $80 \mathrm{~ms}$. Discussion and summary are presented in Sec. 4 . 


\section{Equilibrium Formulation}

We adopt the MHD ordering or the fast ordering and neglect the gyroviscous cancellation (e.g. [12]). The plasma considered here consists of low-temperature high-density electrons (denoted by subscript el), hightemperature low-density electrons (denoted by subscript eh) and low-temperature high-density ions (denoted by subscript i). This model assumes that 1) all three components have isotropic temperatures, 2) the ion fluid is singlyionized, 3) inertia terms are kept for the electron-fluids and 4) equilibrium is axisymmetric. This model is used to represent collisionless ST plasmas sustained by strong RF electron heating.

The model is based on the continuity and force balance equations for the el-electron fluid, the eh-electron fluid and the ion fluid, Ampere's law and Gauss's law for the magnetic field.

$$
\begin{aligned}
& \nabla \cdot\left(n_{\alpha} \mathbf{u}_{\alpha}\right)=0 \quad \text { for } \alpha=\mathrm{el}, \mathrm{eh}, \mathrm{i} \\
& m_{\alpha} n_{\alpha}\left(\mathbf{u}_{\alpha} \cdot \nabla\right) \mathbf{u}_{\alpha}=-\nabla p_{\alpha}+q_{\alpha} n_{\alpha}\left(-\nabla V_{\mathrm{E}}+\mathbf{u}_{\alpha} \times \mathbf{B}\right) \\
& \quad \text { for } \alpha=\mathrm{el}, \mathrm{eh}, \mathrm{i} \\
& \nabla \times \mathbf{B}=\mu_{0} e\left(n_{\mathrm{i}} \mathbf{u}_{\mathrm{i}}-n_{\mathrm{el}} \mathbf{u}_{\mathrm{el}}-n_{\mathrm{eh}} \mathbf{u}_{\mathrm{eh}}\right) \\
& \nabla \cdot \mathbf{B}=0
\end{aligned}
$$

Here $V_{\mathrm{E}}$ is the electrostatic potential, $m_{\mathrm{el}}=m_{\mathrm{eh}}=m_{\mathrm{e}}$ is the electron mass, and standard notations are used for other quantities. We assume the charge-neutrality condition is satisfied such that

$$
n_{\mathrm{i}}=n_{\mathrm{el}}+n_{\mathrm{eh}}
$$

where $q_{\mathrm{el}}=q_{\mathrm{eh}}=-e$ and $q_{\mathrm{i}}=e$ are used.

Using the relations, $\mathbf{u}_{\alpha} \cdot \nabla \mathbf{u}_{\alpha}=\nabla\left(u_{\alpha}^{2} / 2\right)-\mathbf{u}_{\alpha} \times\left(\nabla \times \mathbf{u}_{\alpha}\right)$ and $p_{\alpha}=n_{\alpha} T_{\alpha}$ in Eq. (2), the force balance equation can be written as

$$
\begin{aligned}
& \nabla\left(T_{\alpha}+m_{\alpha} u_{\alpha}^{2} / 2+q_{\alpha} V_{\mathrm{E}}\right)+T_{\alpha} \nabla \ln n_{\alpha}=q_{\alpha} \mathbf{u}_{\alpha} \times \mathbf{\Omega}_{\alpha} \\
& \quad \text { for } \alpha=\text { el, eh, i } \\
& \text { where } \boldsymbol{\Omega}_{\alpha} \equiv \mathbf{B}+\left(m_{\alpha} / q_{\alpha}\right) \nabla \times \mathbf{u}_{\alpha}
\end{aligned}
$$

Note that $q_{\alpha} \boldsymbol{\Omega}_{\alpha}$ is the curl of the generalized momentum for the $\alpha$-species. We call $\boldsymbol{\Omega}_{\alpha}$ the modified magnetic field hereafter. Also note that Eq. (6) is more useful than Eq. (2). In an axisymmetric system, the left side of Eq. (6) has no toroidal component, so no toroidal component should exist on the right side (i.e. $\mathbf{u}_{\alpha} \times\left.\boldsymbol{\Omega}_{\alpha}\right|_{\phi}=0$ ). Therefore we need to consider only two non-toroidal components in Eq. (6). On the other hand, the left and right sides of Eq. (2) have toroidal components $m_{\alpha}\left(\nabla \times \mathbf{u}_{\alpha}\right) \times\left.\mathbf{u}_{\alpha}\right|_{\phi}$ and $q_{\alpha} \mathbf{u}_{\alpha} \times\left.\mathbf{B}\right|_{\phi}$, respectively even in the axisymmetric system. These two terms are canceled in Eq. (6) by using $\boldsymbol{\Omega}_{\alpha}$. Hence we may say that in order to describe the axisymmetric flowing equilibrium, the form of Eq. (6) is more convenient to describe force balance.

\subsection{Dimensionless form}

As dimensionless forms are convenient in numerical computation, we adopt dimensionless variables. The pri- mary scales are (1) $L_{\mathrm{ref}}$ a reference length of a plasma, (2) $I_{\text {ref }}$ a reference plasma current, and (3) $n_{\text {ref }}$ a reference density. These lead to derived scales for the magnetic field $B_{\text {ref }} \equiv \mu_{0} I_{\text {ref }} / L_{\text {ref }}$, velocities $u_{\text {ref }} \equiv B_{\text {ref }} /\left(\mu_{0} m_{\mathrm{i}} n_{\text {ref }}\right)^{1 / 2}$, temperatures $T_{\text {ref }} \equiv m_{\mathrm{i}} u_{\text {ref }}^{2}$, pressures $p_{\text {ref }} \equiv n_{\text {ref }} T_{\text {ref }}$, scalar potential $V_{\text {Eref }} \equiv T_{\text {ref }} / e$, vector potential $A_{\text {ref }} \equiv B_{\text {ref }} L_{\text {ref }}$, poloidal magnetic flux $\psi_{\text {ref }} \equiv B_{\text {ref }} L_{\text {ref }}^{2}$, current density $j_{\text {ref }} \equiv B_{\text {ref }} /\left(\mu_{0} L_{\text {ref }}\right)$, poloidal flow surface function $\Phi_{\text {ref }} \equiv$ $n_{\text {ref }} u_{\text {ref }} L_{\text {ref }}^{2}$ and electric field $E_{\text {ref }} \equiv u_{\text {ref }} B_{\text {ref }}$. Here $m_{\mathrm{i}}$ is the ion mass.

The dimensionless forms of Eqs. (1), (6), (7), (3) and (4) are written as

$$
\begin{aligned}
& \nabla \cdot\left(n_{\alpha} \mathbf{u}_{\alpha}\right)=0 \quad \text { for } \alpha=\mathrm{el}, \mathrm{eh}, \mathrm{i} \\
& \nabla\left(T_{\alpha}+\left(m_{\alpha} / m_{\mathrm{i}}\right) \frac{1}{2} u_{\alpha}^{2}+\left(q_{\alpha} / e\right) V_{\mathrm{E}}\right)+T_{\alpha} \nabla \ln n_{\alpha} \\
& \quad=\left(q_{\alpha} / e\right) \varepsilon^{-1} \mathbf{u}_{\alpha} \times \mathbf{\Omega}_{\alpha} \quad \text { for } \alpha=\mathrm{el}, \mathrm{eh}, \mathrm{i} \\
& \mathbf{\Omega}_{\alpha}=\mathbf{B}+\left(m_{\alpha} / m_{\mathrm{i}}\right)\left(e / q_{\alpha}\right) \varepsilon \nabla \times \mathbf{u}_{\alpha} \quad \text { for } \alpha=\mathrm{el}, \mathrm{eh}, \mathrm{i} \\
& \nabla \times \mathbf{B}=\varepsilon^{-1}\left(n_{\mathrm{i}} \mathbf{u}_{\mathrm{i}}-n_{\mathrm{el}} \mathbf{u}_{\mathrm{el}}-n_{\mathrm{eh}} \mathbf{u}_{\mathrm{eh}}\right) \\
& \nabla \cdot \mathbf{B}=0 \\
& \text { Here } \varepsilon \equiv \ell_{\mathrm{iR}} / L_{\mathrm{ref}} \text { and } \ell_{\mathrm{iR}} \equiv \frac{c}{\sqrt{e^{2} n_{\mathrm{ref}} / \varepsilon_{0} m_{\mathrm{i}}}}
\end{aligned}
$$

$\ell_{\mathrm{iR}}$ is the ion inertial length for the reference density $n_{\mathrm{ref}}$.

\subsection{Surface functions}

Hereafter we adopt the right-hand cylindrical coordinates $(R, \phi, Z)$. Since the magnetic field, $\mathbf{B}$, the mass flow, $n_{\alpha} \mathbf{u}_{\alpha}$, and the modified magnetic field, $\boldsymbol{\Omega}_{\alpha}$, are divergencefree, they can be expressed by the poloidal magnetic flux function $\psi(R, Z)$, the stream function for poloidal flow $\Phi_{\alpha}(R, Z)$ and the function $Y_{\alpha}(R, Z)$, respectively.

$$
\begin{aligned}
& \mathbf{B}=\nabla \psi \times \nabla \phi+R B_{\phi} \nabla \phi \\
& n_{\alpha} \mathbf{u}_{\alpha}=\nabla \Phi_{\alpha} \times \nabla \phi+n_{\alpha} R u_{\alpha \phi} \nabla \phi \quad \text { for } \alpha=\mathrm{el}, \mathrm{eh}, \mathrm{i} \\
& \mathbf{\Omega}_{\alpha}=\nabla Y_{\alpha} \times \nabla \phi+R \Omega_{\alpha \phi} \nabla \phi \quad \text { for } \alpha=\mathrm{el}, \mathrm{eh}, \mathrm{i} \\
& Y_{\alpha}=\psi+\left(m_{\alpha} / m_{\mathrm{i}}\right)\left(e / q_{\alpha}\right) \varepsilon R u_{\alpha \phi} \quad \text { for } \alpha=\mathrm{el}, \mathrm{eh}, \mathrm{i} \\
& \Omega_{\alpha \phi}=B_{\phi}-\left(m_{\alpha} / m_{\mathrm{i}}\right)\left(e / q_{\alpha}\right) \varepsilon R \nabla \cdot\left(\frac{\nabla \Phi_{\alpha}}{n_{\alpha} R^{2}}\right) \\
& \quad \text { for } \alpha=\mathrm{el}, \mathrm{eh}, \mathrm{i}
\end{aligned}
$$

Since $\mathbf{B} \cdot \nabla \psi(R, Z)=0, n_{\alpha} \mathbf{u}_{\alpha} \cdot \nabla \Phi_{\alpha}(R, Z)=0$, and $\boldsymbol{\Omega}_{\alpha} \cdot \nabla Y_{\alpha}(R, Z)=0, \psi(R, Z), \Phi_{\alpha}(R, Z)$ and $Y_{\alpha}(R, Z)$ are surface functions for the magnetic field $\mathbf{B}$, the mass flow $n_{\alpha} \mathbf{u}_{\alpha}$ and the modified magnetic field $\boldsymbol{\Omega}_{\alpha}$, respectively. Equations (17) and (18) are derived from Eq. (10). Note that the deviation $\left(Y_{\alpha}-\psi\right)$ is caused by species toroidal flow velocity while the deviation of $\left(\Omega_{\alpha \phi}-B_{\phi}\right)$ is caused by species poloidal flow velocity. Note also that since the $z$-component of the generalized angular momentum in dimensional form is given by $M_{\alpha z} \equiv R\left(m_{\alpha} u_{\alpha \phi}+q_{\alpha} A_{\phi}\right)$, which 
is conserved in a time-dependent axisymmetric system, $Y_{\alpha}$ defined by Eq. (17) is a dimensionless form of $M_{\alpha z} / q_{\alpha}$.

\subsection{Force balance in directions of $\hat{\phi}, \Omega_{\alpha}$ and $\nabla Y_{\alpha}$}

For the axisymmetric equilibrium considered here, there is no toroidal component in the left side of Eq. (9), so that $\hat{\phi} \cdot\left(\mathbf{u}_{\alpha} \times \mathbf{\Omega}_{\alpha}\right)=0$ yielding $\hat{\phi} \cdot\left(\nabla \Phi_{\alpha} \times \nabla Y_{\alpha}\right)=0$ where $\hat{\phi}$ is the unit vector in the toroidal direction. This condition is satisfied if

$$
\Phi_{\alpha}=\Phi_{\alpha}\left(Y_{\alpha}(R, Z)\right) \quad \text { for } \alpha=\mathrm{el}, \mathrm{eh}, \mathrm{i}
$$

i.e. $\Phi_{\alpha}$ is an arbitrary function depending only on $Y_{\alpha}(R, Z)$. Note that since the inertial term in Eq. (2) has the toroidal component, one cannot use the above mentioned property of the axisymmetric system as long as Eq. (2) is used instead of Eq. (6). Concerning the temperature, we adopt a model that the temperature is an arbitrary function of $Y_{\alpha}(R, Z)$, i.e.

$$
T_{\alpha}=T_{\alpha}\left(Y_{\alpha}(R, Z)\right) \quad \text { for } \alpha=\mathrm{el}, \mathrm{eh}, \mathrm{i}
$$

This model is valid as long as the species thermal speed is much larger that the species poloidal flow velocity. For more detail, see Appendix A of Ref. [3].

The force balance Eq. (9) in the direction of the modified magnetic field $\boldsymbol{\Omega}_{\alpha}$ can be satisfied when the function $F_{\alpha}$ defined below is a function depending only on $Y_{\alpha}(R, Z)$.

$$
\begin{aligned}
& F_{\alpha} \equiv T_{\alpha}\left(Y_{\alpha}\right)\left(1+\ln n_{\alpha}\right)+\left(m_{\alpha} / m_{\mathrm{i}}\right) \frac{1}{2} u_{\alpha}^{2} \\
& +\left(q_{\alpha} / e\right)\left(V_{\mathrm{E}}-C\right)=F_{\alpha}\left(Y_{\alpha}\right) \quad \text { for } \alpha=\text { el, eh, i }
\end{aligned}
$$

where $C$ is an integration constant. Using the above in Eq. (9), the $\nabla Y_{\alpha}$ component of Eq. (9) can be written as

$$
\begin{aligned}
& \varepsilon R\left(F_{\alpha}^{\prime}\left(Y_{\alpha}\right)-T_{\alpha}^{\prime}\left(Y_{\alpha}\right) \ln n_{\alpha}\right) \\
& \quad=\left(q_{\mathrm{e}} / e\right)\left(u_{\alpha \phi}-n_{\alpha}^{-1} \Phi_{\alpha}^{\prime}\left(Y_{\alpha}\right) \Omega_{\alpha \phi}\right) \text { for } \alpha=\mathrm{el}, \mathrm{eh}, \mathrm{i}
\end{aligned}
$$

where the prime denotes differentiation with respect to the argument $Y_{\alpha}$.

\subsection{Ampere's law} comes

Using the expression (14), the left side of Eq. (11) be-

$$
\nabla \times \mathbf{B}=\nabla\left(R B_{\phi}\right) \times \nabla \phi-\hat{\phi}\left\{\frac{\partial}{\partial R}\left(\frac{1}{R} \frac{\partial \psi}{\partial R}\right)+\frac{1}{R} \frac{\partial^{2} \psi}{\partial Z^{2}}\right\}
$$

The toroidal $(\phi)$ component of Ampere's law is written as

$$
\begin{aligned}
& \frac{\partial}{\partial R}\left(\frac{1}{R} \frac{\partial \psi}{\partial R}\right)+\frac{1}{R} \frac{\partial^{2} \psi}{\partial Z^{2}}=-j_{\phi} \quad \text { where } \\
& j_{\phi}=\varepsilon^{-1}\left(n_{\mathrm{i}} u_{\mathrm{i} \phi}-n_{\mathrm{el}} u_{\mathrm{el} \phi}-n_{\mathrm{eh}} u_{\mathrm{eh} \phi}\right)
\end{aligned}
$$

Using Eqs. (15) and (23) in Eq. (11), the poloidal component of Ampere's law is written as

$$
\begin{aligned}
& \nabla\left(R B_{\phi}\right) \times \nabla \phi \\
& \quad=\varepsilon^{-1}\left(\nabla \Phi_{\mathrm{i}}\left(Y_{\mathrm{i}}\right)-\nabla \Phi_{\mathrm{el}}\left(Y_{\mathrm{el}}\right)-\nabla \Phi_{\mathrm{eh}}\left(Y_{\mathrm{eh}}\right)\right) \times \nabla \phi
\end{aligned}
$$

From the above we have

$$
R B_{\phi}=\varepsilon^{-1}\left(\Phi_{\mathrm{i}}\left(Y_{\mathrm{i}}\right)-\Phi_{\mathrm{el}}\left(Y_{\mathrm{el}}\right)-\Phi_{\mathrm{eh}}\left(Y_{\mathrm{eh}}\right)\right)
$$

Defining new functions for the poloidal flows as

$$
\Phi_{\alpha}\left(Y_{\alpha}\right)=-\varepsilon K_{\alpha}\left(Y_{\alpha}\right) \quad \text { for } \alpha=\mathrm{el}, \mathrm{eh}, \mathrm{i}
$$

the toroidal component of the magnetic field can be written as,

$$
B_{\phi}=\left(K_{\mathrm{el}}\left(Y_{\mathrm{el}}\right)+K_{\mathrm{eh}}\left(Y_{\mathrm{eh}}\right)-K_{\mathrm{i}}\left(Y_{\mathrm{i}}\right)\right) / R
$$

Using Eq. (26) in Eg. (22), the species toroidal flow and toroidal current density are written as

$$
\begin{aligned}
u_{\mathrm{i} \phi}= & \varepsilon R\left(F_{\mathrm{i}}^{\prime}\left(Y_{\mathrm{i}}\right)-T_{\mathrm{i}}^{\prime}\left(Y_{\mathrm{i}}\right) \ln n_{\mathrm{i}}\right)-\varepsilon n_{\mathrm{i}}^{-1} K_{\mathrm{i}}^{\prime}\left(Y_{\mathrm{i}}\right) \Omega_{\mathrm{i} \phi} \\
u_{\mathrm{el} \phi}= & -\varepsilon R\left(F_{\mathrm{el}}^{\prime}\left(Y_{\mathrm{el}}\right)-T_{\mathrm{el}}^{\prime}\left(Y_{\mathrm{el}}\right) \ln n_{\mathrm{el}}\right) \\
& -\varepsilon n_{\mathrm{el}}^{-1} K_{\mathrm{el}}^{\prime}\left(Y_{\mathrm{el}}\right) \Omega_{\mathrm{el} \phi} \\
u_{\mathrm{eh} \phi}= & -\varepsilon R\left(F_{\mathrm{eh}}^{\prime}\left(Y_{\mathrm{eh}}\right)-T_{\mathrm{eh}}^{\prime}\left(Y_{\mathrm{eh}}\right) \ln n_{\mathrm{eh}}\right) \\
& -\varepsilon n_{\mathrm{eh}}^{-1} K_{\mathrm{eh}}^{\prime}\left(Y_{\mathrm{eh}}\right) \Omega_{\mathrm{eh} \phi} \\
j_{\phi}= & j_{\mathrm{i} \phi}+j_{\mathrm{el} \phi}+j_{\mathrm{eh} \phi} \\
\text { where } & j_{\mathrm{i} \phi}=\varepsilon^{-1} n_{\mathrm{i}} u_{\mathrm{i} \phi}, j_{\mathrm{el} \phi}=-\varepsilon^{-1} n_{\mathrm{el}} u_{\mathrm{el} \phi} \\
j_{\mathrm{eh} \phi}= & -\varepsilon^{-1} n_{\mathrm{eh}} u_{\mathrm{eh} \phi} \\
j_{\mathrm{i} \phi}= & n_{\mathrm{i}} R\left(F_{\mathrm{i}}^{\prime}\left(Y_{\mathrm{i}}\right)-T_{\mathrm{i}}^{\prime}\left(Y_{\mathrm{i}}\right) \ln n_{\mathrm{i}}\right)-K_{\mathrm{i}}^{\prime}\left(Y_{\mathrm{i}}\right) \Omega_{\mathrm{i} \phi} \\
j_{\mathrm{el} \phi}= & n_{\mathrm{el}} R\left(F_{\mathrm{el}}^{\prime}\left(Y_{\mathrm{el}}\right)-T_{\mathrm{el}}^{\prime}\left(Y_{\mathrm{el}}\right) \ln n_{\mathrm{el}}\right) \\
& +K_{\mathrm{el}}^{\prime}\left(Y_{\mathrm{el}}\right) \Omega_{\mathrm{el} \phi} \\
j_{\mathrm{eh} \phi}= & n_{\mathrm{eh}} R\left(F_{\mathrm{eh}}^{\prime}\left(Y_{\mathrm{eh}}\right)-T_{\mathrm{eh}}^{\prime}\left(Y_{\mathrm{eh}}\right) \ln n_{\mathrm{eh}}\right) \\
& +K_{\mathrm{eh}}^{\prime}\left(Y_{\mathrm{eh}}\right) \Omega_{\mathrm{eh} \phi}
\end{aligned}
$$

$$
\Omega_{\alpha \phi}=B_{\phi}+\left(m_{\alpha} / m_{\mathrm{i}}\right)\left(e / q_{\alpha}\right) \varepsilon^{2} R \nabla \cdot\left(\frac{K_{\alpha}^{\prime}\left(Y_{\alpha}\right) \nabla Y_{\alpha}}{n_{\alpha} R^{2}}\right)
$$$$
\text { for } \alpha=\mathrm{el} \text {, eh, i }
$$

\subsection{Electrostatic potential and densities}

Applying Eq. (21) to the three species, the electrostatic potential $V_{\mathrm{E}}$ is written as

$$
\begin{aligned}
V_{\mathrm{E}} & =\tilde{F}_{\mathrm{i}}-T_{\mathrm{i}}\left(Y_{\mathrm{i}}\right)\left(1+\ln n_{\mathrm{i}}\right)+C \\
& =T_{\mathrm{el}}\left(Y_{\mathrm{el}}\right)\left(1+\ln n_{\mathrm{el}}\right)-\tilde{F}_{\mathrm{el}}+C \\
& =T_{\mathrm{eh}}\left(Y_{\mathrm{eh}}\right)\left(1+\ln n_{\mathrm{eh}}\right)-\tilde{F}_{\mathrm{eh}}+C
\end{aligned}
$$

where the constant $C$ can be used to adjust $V_{\mathrm{E}}$ to the experimentally observed electrostatic potential at a given point and

$$
\tilde{F}_{\mathrm{el}} \equiv F_{\mathrm{el}}\left(Y_{\mathrm{el}}\right)-\frac{1}{2}\left(m_{\mathrm{e}} / m_{\mathrm{i}}\right) u_{\mathrm{el}}^{2}
$$




$$
\begin{aligned}
& \tilde{F}_{\text {eh }} \equiv F_{\text {eh }}\left(Y_{\mathrm{eh}}\right)-\frac{1}{2}\left(m_{\mathrm{e}} / m_{\mathrm{i}}\right) u_{\mathrm{eh}}^{2} \\
& \tilde{F}_{\mathrm{i}} \equiv F_{\mathrm{i}}\left(Y_{\mathrm{i}}\right)-\frac{1}{2} u_{\mathrm{i}}^{2} \\
& u_{\alpha}^{2}=u_{\alpha \phi}^{2}+u_{\alpha p}^{2} \\
& \left(u_{\alpha p}\right)^{2}=\left(\frac{\varepsilon K_{\alpha}^{\prime}\left(Y_{\alpha}\right)}{n_{\alpha} R}\right)^{2}\left\{\left(\frac{\partial Y_{\alpha}}{\partial R}\right)^{2}+\left(\frac{\partial Y_{\alpha}}{\partial Z}\right)^{2}\right\} \\
& \quad \text { for } \alpha=\mathrm{el}, \mathrm{eh}, \mathrm{i}
\end{aligned}
$$

Eliminating the quantity $\left(V_{\mathrm{E}}-C\right)$ from the first and second lines in Eq. (33) yields

$$
\ln n_{\mathrm{i}}+\frac{T_{\mathrm{el}}}{T_{\mathrm{el}}+T_{\mathrm{i}}} \ln \left(1-\frac{n_{\mathrm{eh}}}{n_{\mathrm{i}}}\right)=\frac{\tilde{F}_{\mathrm{el}}+\tilde{F}_{\mathrm{i}}}{T_{\mathrm{el}}+T_{\mathrm{i}}}-1
$$

In the second term on the left-hand side, $n_{\mathrm{el}}=$ $n_{\mathrm{i}}\left(1-n_{\mathrm{eh}} / n_{\mathrm{i}}\right)$ is used. Next, eliminating the quantity $\left(V_{\mathrm{E}}-C\right)$ from the first and third lines in Eq. (33) yields

$$
\ln n_{\mathrm{i}}+\frac{T_{\text {eh }}}{T_{\text {eh }}+T_{\mathrm{i}}} \ln \frac{n_{\mathrm{eh}}}{n_{\mathrm{i}}}=\frac{\tilde{F}_{\mathrm{eh}}+\tilde{F}_{\mathrm{i}}}{T_{\text {eh }}+T_{\mathrm{i}}}-1
$$

Finally eliminating the term $\ln n_{\mathrm{i}}$ from the above two equations, we have the following nonlinear equation for the density ratio $n_{\mathrm{eh}} / n_{\mathrm{i}}$,

$$
\begin{gathered}
\frac{n_{\mathrm{eh}}}{n_{\mathrm{i}}}=\operatorname{Exp}\left\{\frac{\tilde{F}_{\mathrm{eh}}}{T_{\mathrm{eh}}} \frac{\tilde{F}_{\mathrm{el}}+\tilde{F}_{\mathrm{i}}}{T_{\mathrm{el}}+T_{\mathrm{i}}}+\frac{\tilde{F}_{\mathrm{i}} T_{\mathrm{el}}-\tilde{F}_{\mathrm{el}} T_{\mathrm{i}}}{T_{\mathrm{eh}}\left(T_{\mathrm{el}}+T_{\mathrm{i}}\right)}\right. \\
\left.+\frac{\left(T_{\mathrm{eh}}+T_{\mathrm{i}}\right) T_{\mathrm{el}}}{T_{\mathrm{eh}}\left(T_{\mathrm{el}}+T_{\mathrm{i}}\right)} \ln \left(1-\frac{n_{\mathrm{eh}}}{n_{\mathrm{i}}}\right)\right\}
\end{gathered}
$$

The ion density is given by

$$
n_{\mathrm{i}}=\operatorname{Exp}\left\{\frac{\tilde{F}_{\mathrm{el}}+\tilde{F}_{\mathrm{i}}}{T_{\mathrm{el}}+T_{\mathrm{i}}}-1-\frac{T_{\mathrm{el}}}{T_{\mathrm{el}}+T_{\mathrm{i}}} \ln \left(1-\frac{n_{\mathrm{eh}}}{n_{\mathrm{i}}}\right)\right\}
$$

Then, $n_{\mathrm{eh}}=n_{\mathrm{i}} \times\left(n_{\mathrm{eh}} / n_{\mathrm{i}}\right)$ and $n_{\mathrm{el}}=n_{\mathrm{i}}-n_{\mathrm{eh}}$.

Equations (17), (20), (21), (24), (27) - (32) and (35) - (37) constitute a system of equations for axisymmetric equilibrium of a two-electron-temperature plasma. This system includes four second-order partial differential equations and has nine arbitrary functions $T_{\alpha}\left(Y_{\alpha}(R, Z)\right)$, $F_{\alpha}\left(Y_{\alpha}(R, Z)\right)$ and $K_{\alpha}\left(Y_{\alpha}(R, Z)\right)$ for $\alpha=$ el, eh, i. Since these functions determine equilibrium profiles, we call these the profile functions. Next section shows an application of this model which we call the three-fluid model hereafter.

\section{Application to Spherical Torus Plasmas Sustained by Strong RF Electron Heating}

Since the temperature and toroidal flow velocity of the ions, and the temperature and density of the background electrons are measured recently in TST-2 discharge \#115620 where the plasma current is sustained by the LHW (200 MHz) combined with the ECW $(2.45 \mathrm{GHz})$ [13-15]. We apply the present model to reconstruct equi-
Table 1 Parameters of TST-2 shot \#115620 at $80 \mathrm{~ms}$.

$I_{\mathrm{p}}=-8.9 \mathrm{kA}$ with positive $B_{\phi_{-} \text {external }}$

$n_{\mathrm{e}} \approx 0.4 \times 10^{18} \mathrm{~m}^{-3}$

$T_{\mathrm{e}}=11 \pm 3 \mathrm{eV}$ at $R=0.342 \mathrm{~m}$

$u_{\mathrm{i} \phi} \approx 0.5 \mathrm{~km} / \mathrm{s}$ and $u_{\text {ip }}$ is smaller than $u_{\mathrm{i} \phi}$

$T_{\mathrm{i}} \approx 4 \pm 2 \mathrm{eV}$

$n_{\mathrm{e}} \ell \approx 0.1 \times 10^{18} \mathrm{~m}^{-2}$ at $z=0$

librium of this discharge at $80 \mathrm{~ms}$. Table 1 shows the parameters of this shot.

In this computation we choose $L_{\mathrm{ref}}=1[\mathrm{~m}], I_{\mathrm{ref}}=$ $10[\mathrm{kA}]$ and $n_{\text {ref }}=10^{18}\left[\mathrm{~m}^{-3}\right]$ as primary reference scales. Other reference scales are $\varepsilon=0.2278, B_{\text {ref }}=0.0126[\mathrm{~T}]$, $\psi_{\text {ref }}=0.0126[\mathrm{~Wb} / 2 \pi], T_{\text {ref }}=0.78[\mathrm{keV}]$ and $u_{\text {ref }}=$ $274[\mathrm{~km} / \mathrm{s}]$.

\subsection{Computational method}

The numerical algorithm is described in Appendix A. The locations of the magnetic flux loops and the computational domain are shown in Fig. 1. The computational boundary is slightly inside the vacuum vessel. To solve the equation for the magnetic flux function (Step 1 in Appendix A), we use the experimentally observed magnetic flux data on this boundary. Effects of the eddy currents flowing in various structures are excluded from the flux data [16], so the current which is allowed to flow inside the boundary is only the plasma current [8].

Since the slanted parts of the computational boundary (Fig. 1) are chosen to lie on grid points, we adopt the following new coordinates to make the boundary as close as possible to the locations of magnetic flux loops for a given number of grid points. The new coordinates $(X, Y)$ are defined as $X=\sqrt{R}$ and $Y=\tan (1.65 Z)$. To solve the second-order partial differential equations, we use the second-order finite-difference method with equal grid intervals in the $(X, Y)$ coordinates. Maximum numbers of division in the $X$ and $Y$ directions are 128 and 160, respectively. To accelerate numerical computation, a successive over relaxation (SOR) and a progressive multi-grid scheme with four grids are combined. How to select the nine profile functions is crucial in practice. Appendix B shows profile functions used in this computation.

\subsection{Results of reconstructed TST-2 equilib- rium}

Effects of flow appear in two ways. The species toroidal flow makes a difference of $\left(Y_{\alpha}-\psi\right)$ [see Eq. (17)] while the species poloidal flow makes a difference of $\left(\Omega_{\alpha \phi}-B_{\phi}\right)$ [see Eq. (32)]. Figure 1 shows $2 \mathrm{D}$ contours of the magnetic flux function $\psi$ and the surface function $Y_{\mathrm{eh}}$ 


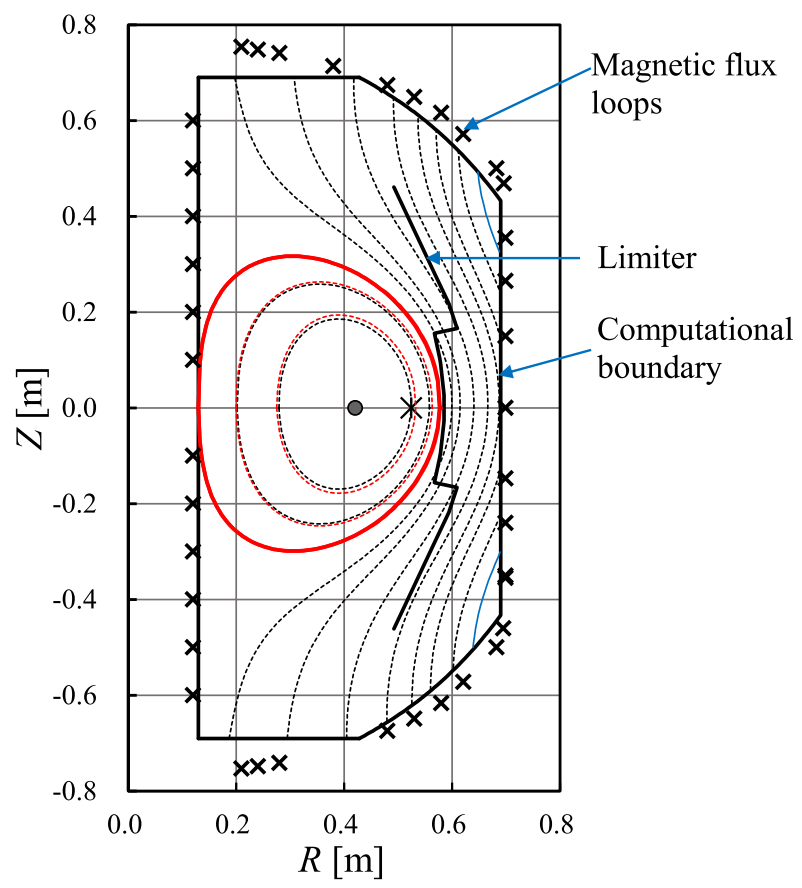

Fig. 1 Flux surfaces of $\psi$ and $Y_{\text {eh. }}$. The red curve represents the LCFS, and the black dotted curves represent flux surfaces outside the LCFS, where the surfaces for $\psi$ and $Y_{\text {eh }}$ are the same. The dotted curves inside the LCFS represent the contours of $\psi$ (black) and $Y_{\text {eh }}$ (red) for the values -0.013 and -0.024 in dimensionless unit. The black circle indicates the position of the magnetic axis and the black asterisk indicates the peak position of the toroidal current density.

of the eh-electron fluid. The red curve represents the last closed flux surface (LCFS) which lies just inside the limiter. To obtain this equilibrium we choose $Y_{\text {icriti }}=Y_{\text {elcriti }}=$ $Y_{\text {ehcriti }}=-0.0053$ in dimensionless unit (see Appendix B). If these critical values are chosen higher than -0.0053 , the LCFS shrinks compared to the red curve while the toroidal current density does not vanish at the outboard limiter. In this equilibrium there is no plasma current density outside the LCFS. This property is different from the previous speculation where appreciable current can flow outside the LCFS (e.g. Ref. [7]) (For more comparison, see Fig. 10). The black dotted curves represent flux surfaces outside the LCFS where the surfaces for $\psi$ and $Y_{\text {eh }}$ are the same because there is no toroidal flow there. The dotted curves inside the LCFS represent the contours of $\psi$ (black) and $Y_{\text {eh }}$ (red) for the values of -0.013 and -0.024 in dimensionless unit. The black circle indicates the position of the magnetic axis $(R=0.421[\mathrm{~m}])$ and the black asterisk indicates the peak position of the toroidal current density $(R=0.524[\mathrm{~m}])$. As the ion and the el-electron toroidal flows are small, differences of $\left(Y_{\mathrm{i}}-\psi\right)$ and $\left(Y_{\mathrm{el}}-\psi\right)$ are very small. Figure 2 shows the density profile for each species on the midplane. The dot-dashed curve, the solid curve and the dotted curve represent the ion fluid density $n_{\mathrm{i}}$, the

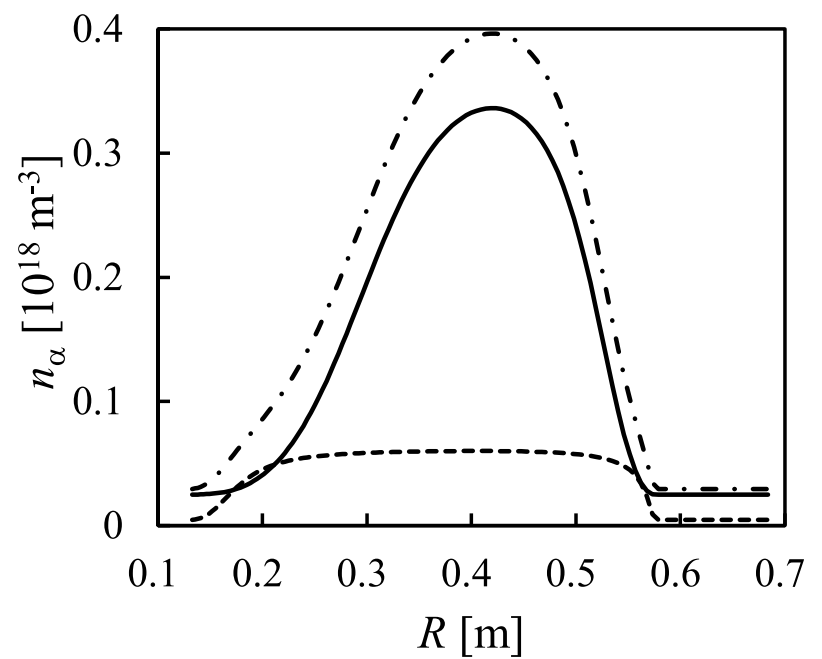

Fig. 2 Midplane density profiles for each species. The dotdashed curve, the solid curve and the dotted curve represent the ion fluid density $n_{\mathrm{i}}$, low energy electron density $n_{\mathrm{el}}$ and high energy electron density $n_{\mathrm{eh}}$, respectively.

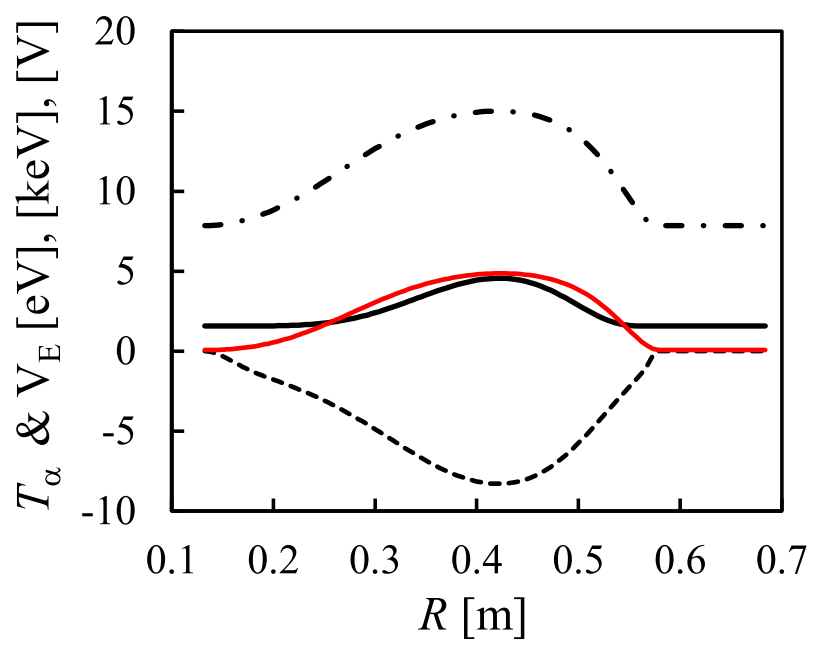

Fig. 3 Midplane temperature profiles of $T_{\mathrm{el}}[\mathrm{eV}]$ (dot-dashed curve), $T_{\mathrm{i}}[\mathrm{eV}]$ (black solid curve), and $T_{\mathrm{eh}}[\mathrm{keV}]$ (red curve). The dotted curve represents $V_{\mathrm{E}}[\mathrm{V}]$.

low energy electron density $n_{\mathrm{el}}$ and the high energy electron density $n_{\mathrm{eh}}$, respectively. Figure 3 shows the midplane temperature profiles of $T_{\mathrm{el}}$ [eV] (dot-dashed curve), $T_{\mathrm{i}}[\mathrm{eV}]$ (black solid curve) and $T_{\mathrm{eh}}[\mathrm{keV}]$ (red curve). The dotted curve represents the electrostatic potential $V_{\mathrm{E}}[\mathrm{V}]$. The midplane profile of the ion toroidal flow velocity $u_{\mathrm{i} \phi}[\mathrm{km} / \mathrm{s}]$ is shown in Fig. 4. Parameters of this reconstructed threefluid equilibrium are summarized in Table 2. Although the ion toroidal flow velocity is slightly higher than the observed value, other parameters are in good agreement with the observed values shown in Table 1 .

Figures 5-9 show interesting features of the reconstructed equilibrium. From Eq. (2) the radial force balance 
Table 2 Parameters of the reconstructed three-fluid equilibrium.

$I_{\mathrm{p}}=-8.9 \mathrm{kA}$ with positive $B_{\phi_{-} \text {external }}$

$n_{\mathrm{e}} \approx 0.4 \times 10^{18} \mathrm{~m}^{-3}$

$T_{\mathrm{e}}=14 \mathrm{eV}$ at $R=0.342 \mathrm{~m}$

$\left|u_{\mathrm{i} \phi}\right|<1.4 \mathrm{~km} / \mathrm{s}$ and $u_{\mathrm{ip}}$ is smaller than $u_{\mathrm{i} \phi}$

$T_{\mathrm{i}} \approx 4.6 \mathrm{eV}$

$n_{\mathrm{e}} \ell \approx 0.08 \times 10^{18} \mathrm{~m}^{-2}$ at $z=0$

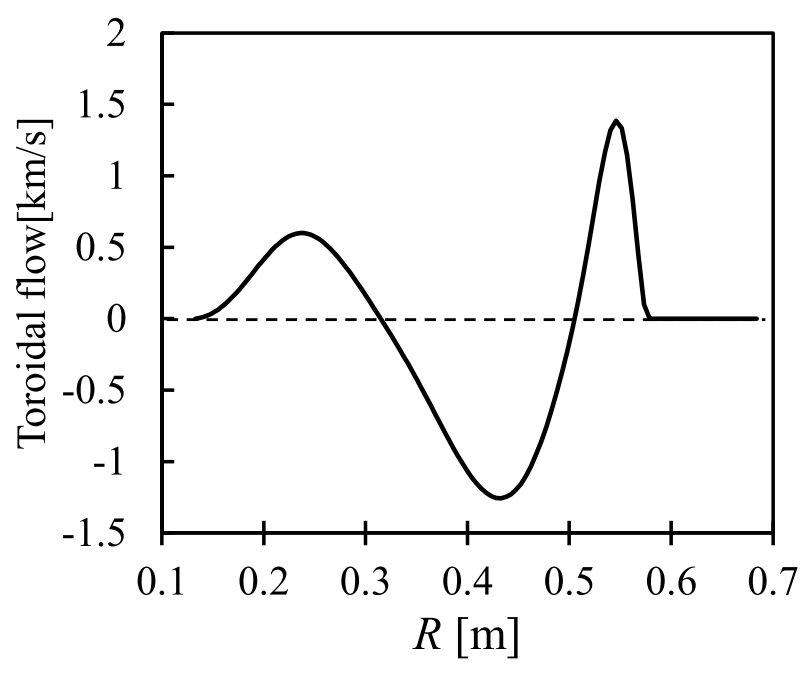

Fig. 4 Midplane ion toroidal flow velocity profile $u_{\mathrm{i} \Phi}[\mathrm{km} / \mathrm{s}]$.

equation is written as,

$$
\begin{aligned}
& -\partial p_{\alpha} / \partial R-q_{\alpha} n_{\alpha} \partial V_{\mathrm{E}} / \partial R+j_{\alpha \phi} B_{Z}+\left(-j_{\alpha Z} B_{\phi}\right) \\
& \quad+m_{\alpha} n_{\alpha}\left(u_{\alpha \phi}^{2} / R-u_{\alpha R} \partial u_{\alpha R} / \partial R-u_{\alpha Z} \partial u_{\alpha R} / \partial Z\right)=0 \\
& \quad \text { for } \alpha=\text { el, eh, i }
\end{aligned}
$$

The 1st, 2nd, 3rd and 4th terms of the above equation represent the pressure gradient force, the electric force, and the Lorentz forces, respectively. The last term comes from the inertial term. In the present case, the last two terms are negligible compared with the centrifugal force, $m_{\alpha} n_{\alpha} u_{\alpha \phi}^{2} / R$. Figure 5 shows the radial force balance on the midplane. The pressure gradient force $-\partial p_{\alpha} / \partial R$ (solid curve), the electric force (dotted curve), the Lorentz forces $j_{\alpha \phi} B_{Z}$ (dashed curve), $-j_{\alpha Z} B_{\phi}$ (dot-dashed curve), and the centrifugal force (2-dot-dashed curve) are shown. In the ion fluid force balance, the pressure gradient force and the electric force dominate (Fig.5(a)). As a result, the ion fluid is confined by the electric force. The other three forces acting on the ion fluid are too small to see. For the eh-electron fluid, the pressure gradient force and the Lorentz forces $j_{\mathrm{eh} \phi} B_{Z}$ are balanced (Fig. 5(c)). The other three forces acting on the eh-electron fluid are too small to see. For the el-electron fluid, the sum of the pressure gradient force and the electric force keep balance with the

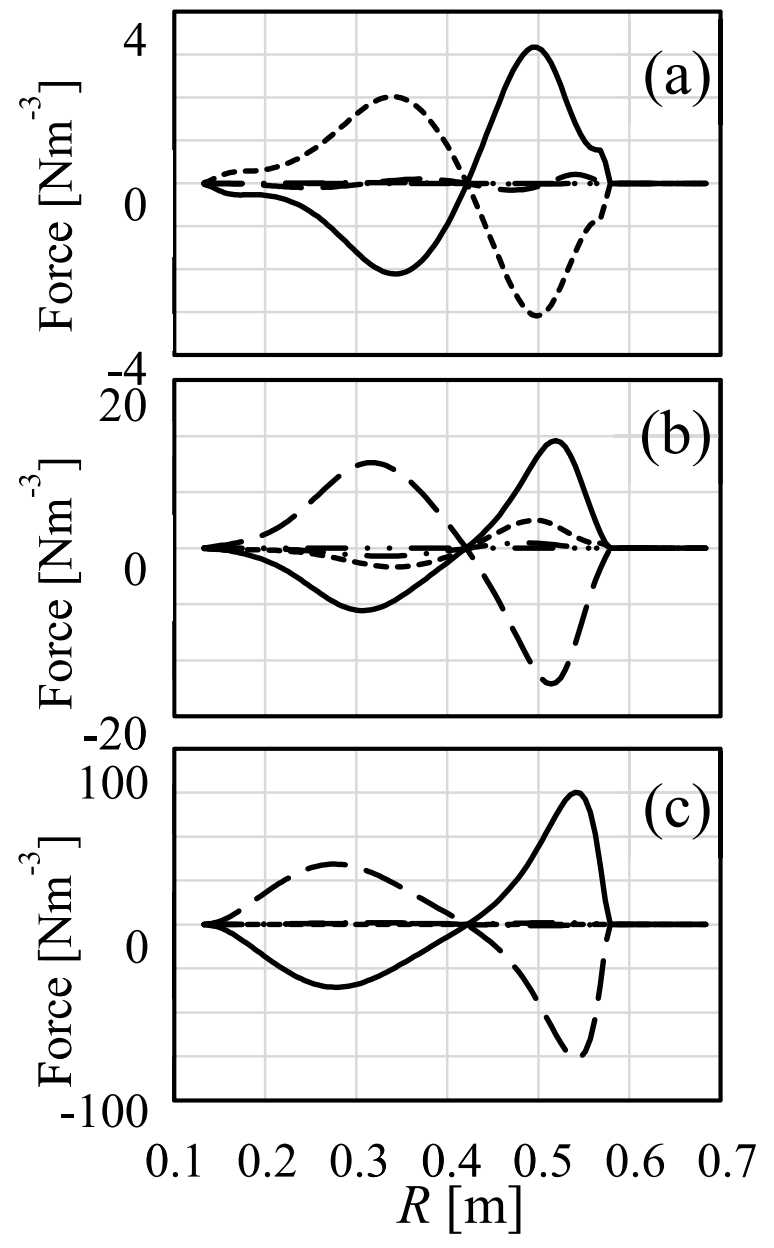

Fig. 5 Radial forces for the ion fluid (a), the low energy electron fluid (b) and the high energy electron fluid (c). The pressure gradient force $-\partial p_{\alpha} / \partial R$ (solid curve), the electric force (dotted curve), the Lorentz force $j_{\alpha \Phi} B_{Z}$ (dashed curve), $-j_{\alpha Z} B_{\Phi}$ (dot-dashed curve), the centrifugal force (2-dot-dashed) are shown.

Lorentz force $j_{\mathrm{el} \phi} B_{Z}$ (Fig. 5(b)). The other two forces acting on the el-electron fluid are too small to see. Note that the scales of the vertical axis in (a), (b) and (c) are quite different. The force balance mentioned above is complex and its features could not be described by the standard onefluid MHD. Figure 6 shows the species toroidal current density profile on the midplane. The solid, the dot-dashed, the dashed and the dotted curves represent $j_{\phi}, j_{\mathrm{eh} \phi}, 10 \times j_{\mathrm{el} \phi}$, and $100 \times j_{\mathrm{i} \phi}$, respectively. Nearly the entire current is carried by the eh-electron (high energy low density electron) fluid. Figure 7 shows the species pressure profile on the midplane. The solid, the dot-dashed, the dashed and the dotted curves represent the total pressure $p=p_{\mathrm{eh}}+p_{\mathrm{el}}+p_{\mathrm{i}}$, $p_{\text {eh }}, 10 \times p_{\text {el }}$ and $10 \times p_{\mathrm{i}}$, respectively. The pressure is dominated by that of the eh-electron fluid. Figure 8 shows the ratios of the toroidal flow velocity to the thermal velocity for each species fluid. The solid, the dashed and the dotted curves represent $10 \times\left|u_{\mathrm{i} \phi} / C_{\mathrm{S}}\right|, 10 \times\left|u_{\mathrm{el} \phi} / v_{\text {th_el }}\right|$ and $\left|u_{\mathrm{eh} \phi} / v_{\text {th_eh }}\right|$, respectively. Here $C_{\mathrm{S}}=\left(\left(T_{\mathrm{i}}+T_{\mathrm{el}}\right) / m_{\mathrm{i}}\right)^{1 / 2}$ is 


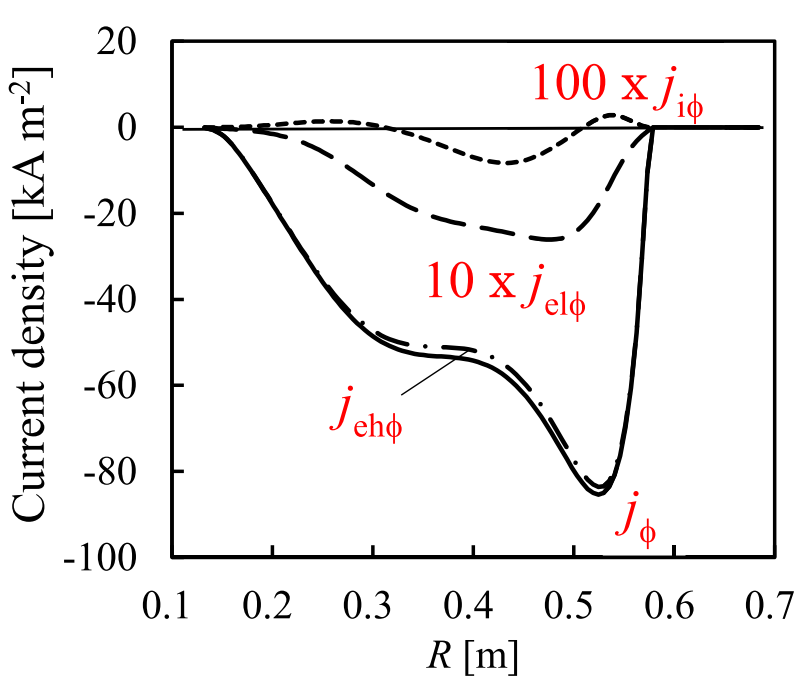

Fig. 6 Midplane toroidal current density profiles. The solid, the dot-dashed, the dashed and the dotted curves represent $j_{\Phi}, j_{\mathrm{eh} \Phi}, 10 \times j_{\mathrm{el} \Phi}$, and $100 \times j_{\mathrm{i} \Phi}$, respectively.

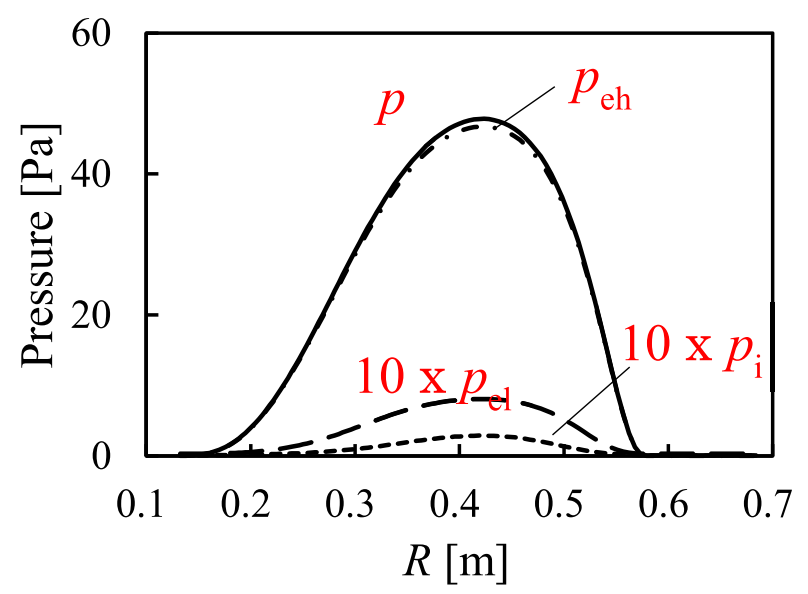

Fig. 7 Midplane pressure profiles. The solid curve represents the total pressure $p=p_{\mathrm{eh}}+p_{\mathrm{el}}+p_{\mathrm{i}}$, while the dot-dashed, the dashed and the dotted curves represent $p_{\mathrm{eh}}, 10 \times p_{\mathrm{el}}$, and $10 \times p_{\mathrm{i}}$, respectively.

the sound speed. The ratio of the toroidal flow to the thermal velocity of the eh-electron fluid is very large near the outboard boundary. In this equilibrium, the vertical current density $j_{Z}$ is about $0.1 \%$ of the toroidal current density $j_{\phi}$. This means that not only the toroidal current density but also the toroidal flow velocity is dominated by the terms proportional to the radial coordinate $R$ (Eqs. (28) and (31)). This makes the current density profile (Fig. 6) different from the temperature and pressure profiles (Figs. 3 and 7 ). This is the reason why the peak position of the toroidal current density shifts outward compared with the position of the magnetic axis (Fig. 1). Asymmetry in the ratio of the toroidal flow to the thermal velocity of each fluid can be explained by the reason mentioned above. Figure 9 shows the midplane profiles of the magnetic flux function $\psi$ and the

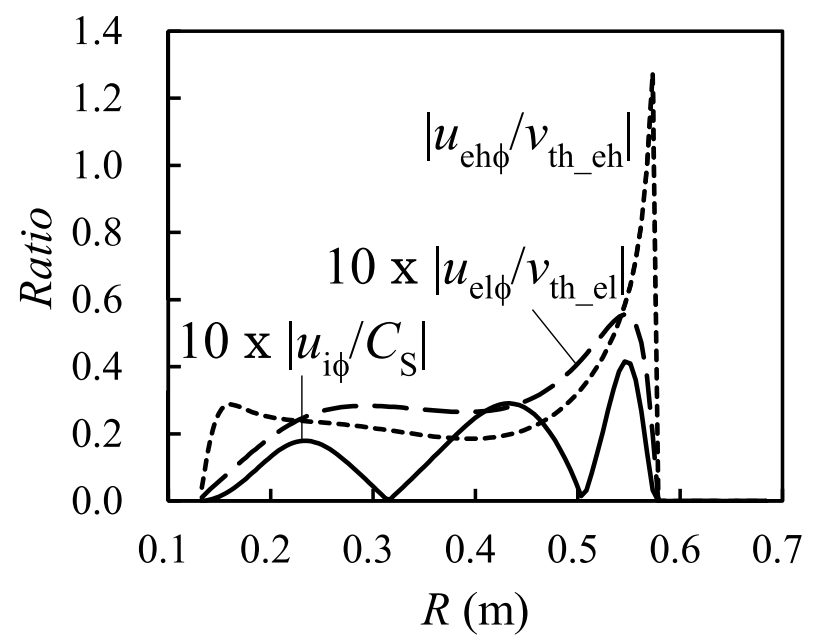

Fig. 8 Ratios of the toroidal flow velocity to the thermal velocity for each species. The solid, the dashed and the dotted curves represent $10 \times\left|u_{\mathrm{i} \Phi} / C_{\mathrm{S}}\right|, 10 \times\left|u_{\mathrm{el} \Phi} / v_{\text {th_el }}\right|$ and $\left|u_{\mathrm{eh} \Phi} / v_{\text {th_eh }}\right|$, respectively. Here $C_{\mathrm{S}}=\left(\left(T_{\mathrm{i}}+T_{\mathrm{el}}\right) / m_{\mathrm{i}}\right)^{1 / 2}$.

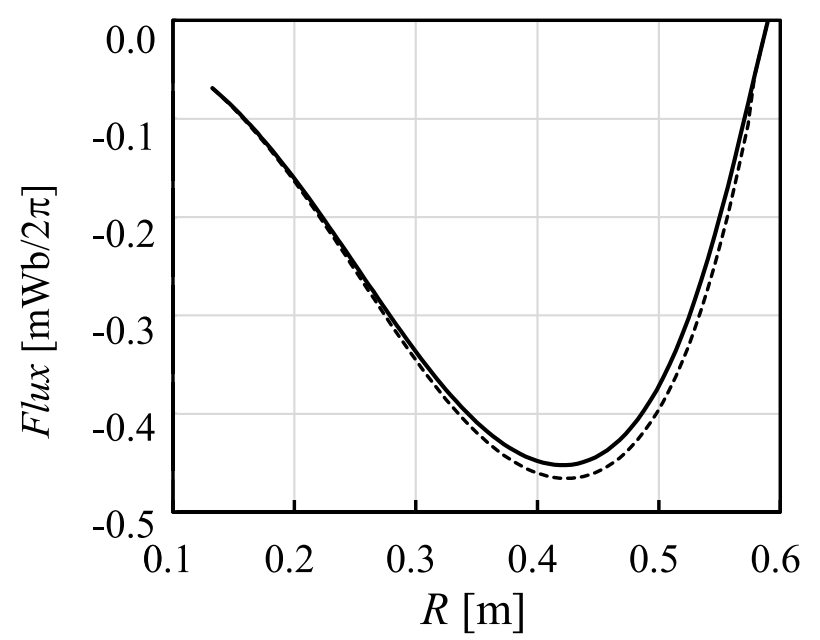

Fig. 9 Magnetic flux function $\psi$ (solid curve) and the flux function $Y_{\text {eh }}$ (dotted curve) for high temperature electron fluid on the midplane.

flux function $Y_{\mathrm{eh}}$ of the eh-electron fluid. As the maximum of $u_{\mathrm{eh} \phi}$ is about $1 / 3$ of the speed of light, the difference of $Y_{\mathrm{eh}}-\psi=-\left(m_{\mathrm{e}} / m_{\mathrm{i}}\right) \varepsilon R u_{\mathrm{eh} \phi}$ is somewhat significant. As mentioned above, the species poloidal flow makes the difference of $\left(\Omega_{\alpha \phi}-B_{\phi}\right)$ [see Eq. (32)]. Since the poloidal flow is small in this equilibrium, $\left|\left(\Omega_{\mathrm{eh} \phi}-B_{\phi}\right) / B_{\phi}\right|<3 \times 10^{-5}$, this difference is very small.

Figure 10 shows a comparison of the magnetic flux contours of the present three-fluid equilibrium (black solid curves) and those of the standard Grad-Shafranov equilibrium (red dotted curves). Contours outside the LCFS (see Fig. 1) are in good agreement. Since the same boundary magnetic flux data are used in these two equilibria, this coincidence is a natural result. Contours inside the LCFS are 


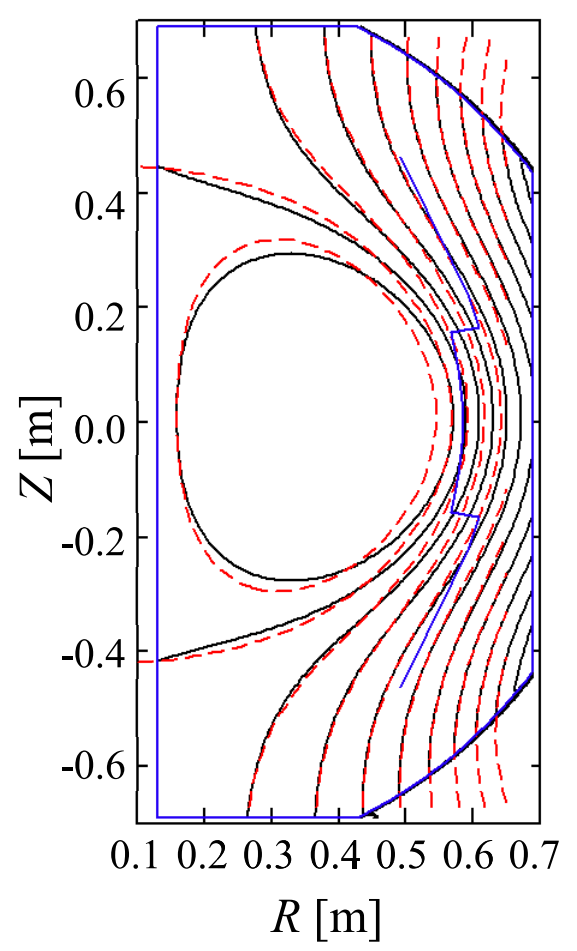

Fig. 10 Comparison of the magnetic flux function of the threefluid equilibrium (black) with that of the standard GradShafranov equilibrium (red).

model-dependent. Inside the LCFS contours of the threefluid equilibrium are slightly expanded in the radial direction compared with those of the standard Grad-Shafranov equilibrium.

\section{Discussion and Summary}

In order to describe a solenoid-free RF sustained ST plasma, a three-fluid equilibrium model has been developed. In this model the el-electron fluid represents a lowtemperature, high-density electron component, and the ehelectron fluid represents a high-temperature, low-density electron component. Equilibrium equations consist of a nonlinear algebraic equation for density ratio, four 2 nd order partial differential equations (PDEs) and some auxiliary algebraic equations. One of PDEs is a equation for the poloidal magnetic flux function $\psi$ and the other three PDEs are equations for the function $Y_{\alpha}(\alpha=\mathrm{el}, \mathrm{eh}, \mathrm{i})$. Note that the highest order derivatives arises from the poloidal magnetic flux and the species poloidal mass flow.

The model was applied for the first time to reconstruct the TST-2 RF sustained discharge \#115620 at $80 \mathrm{~ms}$. The parameters of the reconstructed three-fluid equilibrium (Table 2) are in good agreement with the experimental data (Table 1) for this shot. The interesting features of this equilibrium are shown in Figs. 1-9. It is found that (1) there is no plasma current density outside the LCFS, (2) the toroidal current density and pressure are dominated by the eh-electron fluid, and (3) the radial force balance is quite different among the three fluid species, i.e. the ion fluid is confined by the electric force arising from the negative electrostatic potential while the pressure gradient force of the eh-electron fluid is balanced by its Lorentz force $j_{\mathrm{eh} \phi} B_{Z}$. These results are different from previous speculations. Further investigation on this discrepancy will be our future work.

\section{Acknowledgments}

One of the authors (A.I.) would like to thank Dr. Y.-K. Martin Peng for excellent guidance to the research area of solenoid-free RF-sustained ST plasmas and for discussion on electrostatic potential.

\section{Appendix A. Numerical Algorithm}

In this three-fluid model, there are four partial differential equations (PDEs), one is for $\psi$ [Eq. (24)] and the others are for $Y_{\alpha}$ [Eq. (32) with Eq. (28) and Eq. (17)]. In a general case, we must solve these four PDEs simultaneously with the help of algebraic equations for densities. Since the toroidal flow velocity vanishes on the boundary, the realistic boundary conditions for $\psi$ and $Y_{\alpha}$ should be the same.

As shown in Sec. 3, the toroidal flow velocity $u_{\alpha \phi}$ is not large implying that $Y_{\alpha} \approx \psi$ in the leading order approximation. Since the poloidal flow velocity $u_{\alpha \text { _poloidal }}$ is also small in the present analysis, $\Omega_{\alpha \phi} \approx B_{\phi}$ in the leading order approximation. This means that the second term of Eq. (32) is a small correction term of $B_{\phi}$. In this circumstance, we can treat the second term of Eq. (32) as a perturbation of $B_{\phi}$. Therefore, we adopt the following iteration procedure to obtain self-consistent solutions where all the PDEs are solved with sufficient accuracy. In practice this algorithm is very efficient and the boundary conditions for the toroidal and poloidal flows are well satisfied.

(Step 0): Prepare approximate values of $Q$ and $n_{\mathrm{eh}} / n_{\mathrm{i}}$. (Step 1): Update $\psi$ for a prescribed $Q$

$$
\begin{aligned}
& R^{2} \nabla \cdot\left(\frac{\nabla \psi}{R^{2}}\right)=Q \quad \text { where } \\
& Q \equiv-R j_{\phi}=-R\left(j_{\mathrm{i} \phi}+j_{\mathrm{el} \phi}+j_{\mathrm{eh} \phi}\right) .
\end{aligned}
$$

The species toroidal current density is given by Eq. (31). (Step 2): Update $Y_{\alpha}(R, Z)$ of Eq. (17), $B_{\phi}$ of Eq. (27), $\Omega_{\alpha \phi}$ of Eq. (32) and $u_{\alpha \phi}$ of Eq. (28), because $\psi$ was updated.

(Step 3): Update $u_{\alpha \phi}, Y_{\alpha}(R, Z)$ and $B_{\phi}$ again.

(Step 4): Solve the ratio $n_{\mathrm{eh}} / n_{\mathrm{i}}$ iteratively for given other quantities.

(Step 5): Update $n_{\mathrm{i}}, n_{\mathrm{eh}}, n_{\mathrm{el}}$.

(Step 6): If convergence is not sufficient, return to Step 1 to iterate.

\section{Appendix B. Profile Functions}

Various profile functions were tested. The following functions were found to work well. 


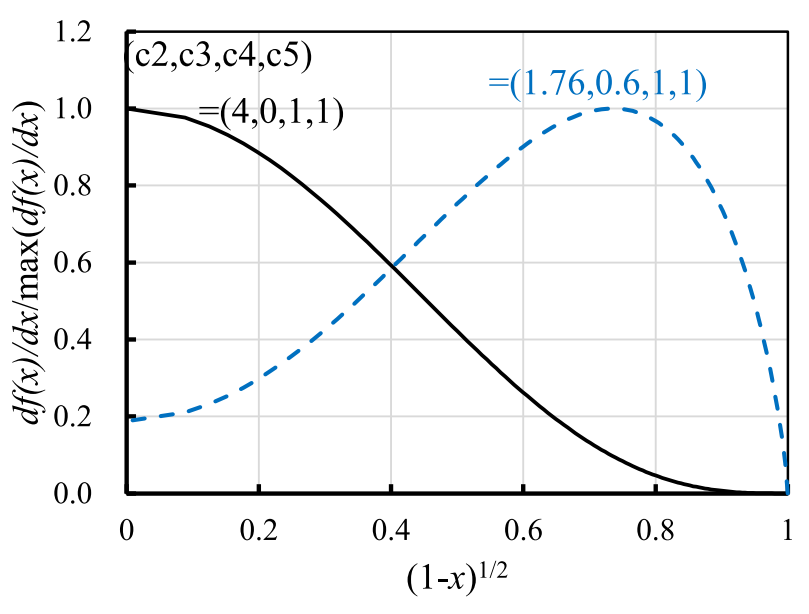

Fig. A1 $\mathrm{d} f / \mathrm{d} x$ as a function of $(1-x)^{1 / 2}$. The black solid and the blue dashed curves represent peaked and hollow profiles, respectively.

For $\alpha=$ el, eh, i

$T_{\alpha}\left(Y_{\alpha}\right)=\left(\begin{array}{ll}C_{T \alpha 0}+C_{T \alpha 1} f\left(-\left(Y_{\alpha}-Y_{\alpha \text { crit }}\right)\right. & \\ \left.C_{T \alpha 2}, C_{T \alpha 3}, C_{T \alpha 4}, C_{T \alpha 5}\right) & \text { if } Y_{\alpha}-Y_{\alpha \text { crit }} \leq 0 \\ C_{T \alpha 0} & \text { if } Y_{\alpha}-Y_{\alpha \text { crit }}>0\end{array}\right.$

$K_{\alpha}\left(Y_{\alpha}\right)=\left(\begin{array}{ll}C_{K \alpha 0}+C_{K \alpha 1} f\left(-\left(Y_{\alpha}-Y_{\alpha \text { crit }}\right)\right. & \\ \left.C_{K \alpha 2}, C_{K \alpha 3}, C_{K \alpha 4}, C_{K \alpha 5}\right) & \text { if } Y_{\alpha}-Y_{\alpha \text { crit }} \leq 0 \\ C_{K \alpha 0} & \text { if } Y_{\alpha}-Y_{\alpha \text { crit }}>0\end{array}\right.$

$F_{\alpha}\left(Y_{\alpha}\right)=\left(\begin{array}{ll}C_{F \alpha 0}+C_{F \alpha 1} f\left(-\left(Y_{\alpha}-Y_{\alpha \text { crit }}\right) ;\right. & \text { if } Y_{\alpha}-Y_{\alpha \text { crit }} \leq 0 \\ \left.C_{F \alpha 2}, C_{F \alpha 3}, C_{F \alpha 4}, C_{F \alpha 5}\right) & \text { if } Y_{\alpha}-Y_{\alpha \text { crit }}>0\end{array}\right.$

where $Y_{\alpha \text { crit }}$ is a constant and the generic function $f$ and its derivatives are given by

$$
\begin{gathered}
\begin{aligned}
f\left(x ; c_{2}, c_{3}, c_{4}, c_{5}\right) & \equiv\left(x / c_{4}\right)^{c_{2}}\left[1-c_{3}\left(x / c_{4}\right)^{c_{5}}\right] \\
\equiv & f\left(0, x, c_{2}, c_{3}, c_{4}, c_{5}\right)
\end{aligned} \\
\begin{aligned}
\mathrm{d} f\left(x ; c_{2}, c_{3}, c_{4}, c_{5}\right) / \mathrm{d} x \\
=
\end{aligned} \\
\equiv\left(c_{2} / c_{4}\right)\left(x / c_{4}\right)^{c_{2}-1}\left[1-c_{3}\left(1+c_{5} / c_{2}\right)\left(x / c_{4}\right)^{c_{5}}\right] \\
\frac{\mathrm{d}^{2} f\left(x ; c_{2}, c_{3}, c_{4}, c_{5}\right)}{\mathrm{d} x^{2}} \\
=\frac{c_{2}\left(c_{2}-1\right)}{c_{4}^{2}}\left(x / c_{4}\right)^{c_{2}-2} \\
\cdot\left[1-c_{3}\left(1+\frac{c_{5}}{c_{2}}\right)\left(1+\frac{c_{5}}{c_{2}-1}\right)\left(x / c_{4}\right)^{c_{5}}\right] \\
\equiv f\left(2, x, c_{2}, c_{3}, c_{4}, c_{5}\right)
\end{gathered}
$$

Note that $\mathrm{d}^{2} f / \mathrm{d} x^{2}$ is used only in the $K_{\alpha}$ profile function. If $c_{2}>1$, the functions $f$ and $\mathrm{d} f / \mathrm{d} x$ are zero when $x=$ 0 . We use this property in selecting the profile functions. Examples of the profile $\mathrm{d} f / \mathrm{d} x$, which is related to flow (see Eq. (28)) are shown in Fig. A1. For the horizontal axis, we use $(1-x)^{1 / 2}$ which approximates the minor radius when $x$ is expressed by a parabolic function of the major radius $R$.

[1] L.C. Steinhauer, Phys Plasmas 6, 2734 (1999).

[2] A. Thyagaraja and K.G. McClements, Phys. Plasmas 13, 062502 (2006).

[3] A. Ishida, L.C. Steinhauer and Y.-K.M. Peng, Phys. Plasmas 17, 122507 (2010).

[4] A. Ishida and L.C. Steinhauer, Phys. Plasmas 19, 102512 (2012).

[5] A. Ishida, C.O. Harahap, L.C. Steinhauer and Y.-K.M. Peng, Phys. Plasmas 11, 5197 (2004).

[6] A. Ishida, C.Z. Chen and Y.-K.M. Peng, Phys. Plasmas 12, 052113 (2005).

[7] Y. Takase, Y. Fukuda, X. Gao et al., J. Plasma Fusion Res. 8, 719 (2002).

[8] Y.-K.M. Peng, A. Ishida, Y. Takase, A. Ejiri, N. Tsujii, T. Maekawa, M. Uchida, H. Zushi, K. Hanada and M. Hasegawa, Plasma Fusion Res. 9, 3403146 (2014).

[9] A. Ejiri, Y. Takase, T. Oosako, T. Yamaguchi, Y. Adachi, O. Watanabe, Y. Nagashima, B.I. An, H. Kobayashi, H. Kurashina, H. Hayashi, H. Matsuzawa, K. Yamada, H. Tojo, T. Masuda, M. Sasaki, R. Kumazawa, H. Kasahara and F. Shimpo, Nucl. Fusion 49, 065010 (2009).

[10] A. Ejiri, Y. Takase, H. Kasahara, T. Yamada, K. Hanada, K.N. Sato, H. Zushi, K. Nakamura, M. Sakamoto, H. Idei, M. Hasegawa, A. Iyomasa, N. Imamura, K. Esaki, M. Kitaguchi, K. Sasaki, H. Hoshika, O. Mitarai and N. Nishino, Nucl. Fusion 46, 709 (2006).

[11] W.D. Jones, A. Lee, S.M. Gleman and H.J. Doucet, Phys. Rev. Lett. 35, 1349 (1975).

[12] J.J. Ramos, Phys. Plasmas 12, 052102 (2005).

[13] Y. Takase, A. Ejiri, H. Kakuda, T. Oosako, T. Shinya, T. Wakatsuki, T. Ambo, H. Furui, T. Hashimoto, J. Hiratsuka, H. Kasahara, K. Kato, R. Kumazawa, C.P. Moeller, T. Mutoh, A. Nakanishi, Y. Nagashima, K. Saito, T. Sakamoto, T. Seki, M. Sonehara, R. Shino, H. Togashi, O. Watanabe and T. Yamaguchi, Nucl. Fusion 53, 063006 (2013).

[14] T. Shinya, Y. Takase, C.P. Moeller, T. Wakatsuki, T. Inada, T. Oosako, H. Kakuda, A. Ejiri, N. Tsujii, H. Furui, J. Hiratsuka, K. Imamura, K. Nakamura, A. Nakanishi, M. Sonehara, H. Togashi, S. Tsuda and T. Yamaguchi, Plasma Fusion Res. 9, 1202133 (2014).

[15] S. Tsuda, A. Ejiri, H. Tanaka, Y. Takase, M. Uchida, T. Maekawa, N. Tsujii and T. Takeuchi, Plasma Fusion Res. 10, 1202064 (2015).

[16] L.L. Lao et al., Nucl. Fusion 25, 1611 (1985). 